\title{
Sequential autologous fat injection techniques for volumetric face lifting in young Korean females
}

\author{
Kyoungjin Kang, MD, PhD \\ Seoul Cosmetic Surgery Clinic, Busan, Rep. of Korea
}

\begin{abstract}
Background: Various fat grafting techniques have been preferentially performed to restore facial volume deficiency. However, no reports have focused on simultaneous volume deficiency and sagging appearance improvements, barring the sequential autologous fat injection (SAFI) technique.

Objective: This study aimed to show real evidence of volumetric lifting using the SAFI technique.

Methods: From 2007 to 2009, we performed the SAFI technique on 10 patients in their 20s. Fat was purified using Coleman's method and sequentially injected into each defective region from the superolateral to the anteroinferior direction. The fat was placed subsequently in the supra-periosteal, deep, and superficial layers (approximately $60 \%, 30 \%$, and $10 \%$ of the injected volume, respectively) of each region to increase the skeletal support for the soft tissue. Morphological changes were evaluated by comparing pre- and postoperative photographs. The effects were monitored from 3 months to 1.5 years.

Results: We included 10 patients with an average age of $25.4 \pm 2.56$ years, having relatively small and flat malar bones with negative globe-to-skeletal rim relations; moreover, they were injected averagely with $38.8 \pm 4.89 \mathrm{ml}$ of fat. In most of the cases, the facial contour changed from a rectangular to an oval shape. Furthermore, the appearance was lifted through great volume restoration in the midface and volume reduction in the deep nasolabial folds (NLFs) and jowls. The wrinkle severity rating scale (WSRS) grade decreased in 7 patients ( 3 on both sides and 4 on either side) and increased in 3 patients. The WSRS improvement was not statistically significant; however, NLFs showed a younger, more natural, and lifted look in all cases. Patient satisfaction was relatively high (4.4 \pm 0.5$)$.

Conclusion: The results indicated that the SAFI technique could be used for both volume deficiency and sagging appearance improvements because it preferentially focused on both skeletal deformity and soft tissue defect corrections.
\end{abstract}

Keywords: face lifting; face sagging; fat grafting; skeletal deformity; volume deficiency

\section{Introduction}

Fat grafting, including structural fat grafting [1], fat rebalancing [2], fat autologous muscular injection [3], complement fat grafting [4], and sequential autologous fat injection (SAFI) [5], has been widely performed as a conventional method for facial and corporal volume restoration. Among the different fat graft- ing techniques, SAFI is known to function on a concept of volumetric lifting using fat grafting.

Generally, small zygomatic bones and aging manifestations such as deep nasolabial folds (NLFs) and sagging jowls are seen in both young and old patients. This is mainly due to the decreased skeletal support of the soft tissue. An allograft implant is a hard material used to correct skeletal deformity. Herein, the 
author tried to inject fat to correct skeletal deformity in young patients who had complained of volume deficiency and sagging appearance. The fat was mainly placed in the supra-periosteal layer including the retaining ligaments. Thereafter, different volumes of fat were sequentially injected into the deep and superficial fat layers (the deeper layer the more injected fat volume) using the SAFI technique. This hypothesis is supported by some studies that reported that facial sagging was caused not only by soft tissue aging but also by skeletal deformities, and therefore can be improved by volume modification and restoration, preferentially focused on the bone tissues $[6,7]$.

Thus, the author hypothesized that the SAFI technique is the fundamental solution to improve both skeletal deformity and soft tissue deficiency. It is expected to produce not only volume restoration but also volumetric lifting by increasing skeletal support.

To evaluate the effect of the SAFI technique, I assessed the globe-to-skeletal rim relations [8], wrinkle severity rating scale (WSRS) [9] grade, Ogee curve [10], and change of regional contour from the pre- and postoperative patient photographs.

\section{Materials and methods}

The fat harvesting and purification procedures were performed using a modified version of Coleman's method [1]. When we inject the fat (100\%) to the soft tissue, among them, $60 \%$ is injected for supraperiosteal layer for increasing skeletal support for the soft tissue (by attaching the fat to the bone, it makes the bone be larged and protrude anteriorly), both $30 \%$ for deep fat layer and $10 \%$ for superficial fat layer is for restoration of soft tissue volume. So, there are two functions, one is for increasing skeletal support and restoring soft tissue volume. In the area without the bone, the fat was attached to the muscle fascia or injected into the deep fat pad. Moreover, the fat was injected in a regular order as shown in Fig. 1 [5]. Grafting was performed under local anesthesia coupled with intravenous sedation using propofol.

\section{Results}

This study included 10 females with an average age of $25.4 \pm 2.56$ years. All participants had negative globe-to-skeletal rim relations. An average of $38.8 \pm 4.89 \mathrm{ml}$ of purified fat was grafted, and the midfacial regions of 9, 10, 11, 13, 14 (Table 1) were most frequently grafted for all 10 patients. The target volumes of infraorbital hollowness, mid-cheek furrow, and cheek depression were successfully attained by fat grafting to these areas (Fig. 2-4).

Of the 10 patients, WSRS grade decreased in 3 patients (cases 3,6 , and 7), and only 4 showed a decrease in either the right or left side grades (cases 1, 2, 4, and 5). The WSRS grade increased in 3 patients (cases 8, 9, and 10) (Table 1). However, the average WSRS (right/left) grades were $2.3 \pm 0.46 / 2.0 \pm 0.89$ and $2.1 \pm 0.53 / 1.7 \pm 0.46$ before and after fat grafting, respectively, with no statistically significant improvement $(\mathrm{p}<0.05)$.

Three patients with increased WSRS grades (cases 8-10) had significant volume reduction in the maxillary part of the midface. Postoperatively, the NLF deepened due to an advanced anterior projection following fat grafting. However, it appeared more natural and harmonized than before the procedure (Fig. 4). Patient satisfaction was relatively high at $4.4 \pm 0.5$.

The facial contours changed from rectangular to oval in 6 females (cases 1, 3, 4, 7, 9, and 10) and from an unbalanced to a balanced oval shape in 4 females (cases 2, 5, 6, and 8).

In all cases, we observed a series of arcs and convexities with a homogenized and balanced transition without demarcation (Fig. 2-4).

Anterior projection by fat grafting in the midface led to a volume reduction in the folded part of the NLF, which tightened the overlying skin, leading to softening of the folds (cases 1-7,

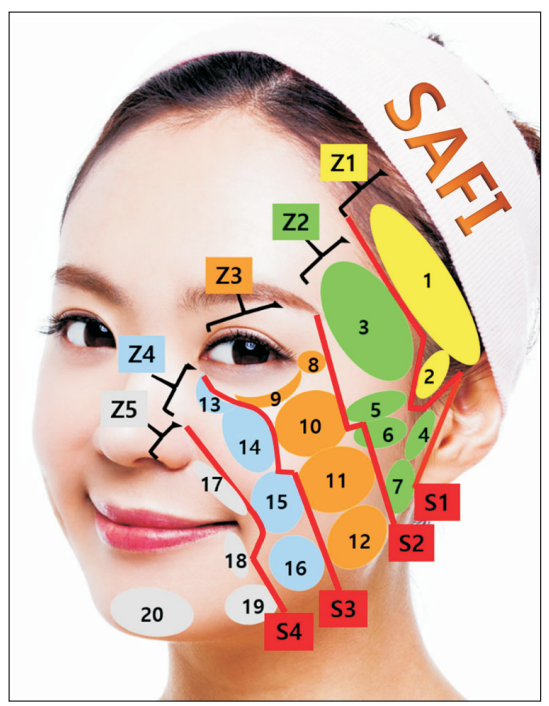

Fig. 1. Lines, zones, and possible regions of SAFI. The face is divided into 5 zones by 4 SAFI lines. The fat is injected in order from zone 1 to 5 , and from the upper to the lower region in each zone. In each region, the fat is injected into the deep, middle, and superficial layers in that order. The numbers ( 1 to 20) represent the order of injections. SAFI, sequential autologous fat injection; Z1-5, injected zone; S1-4, SAFI lines. 


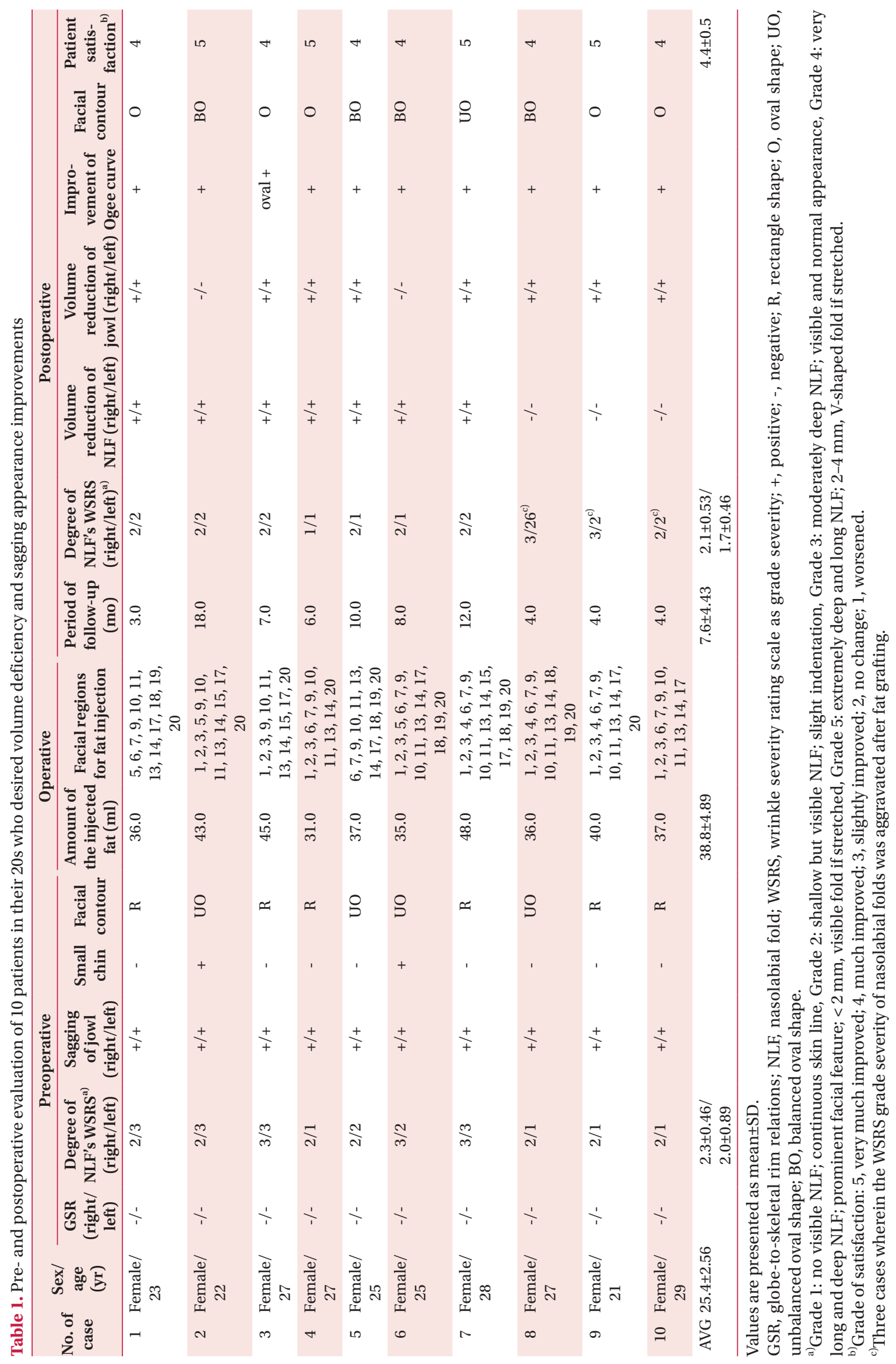



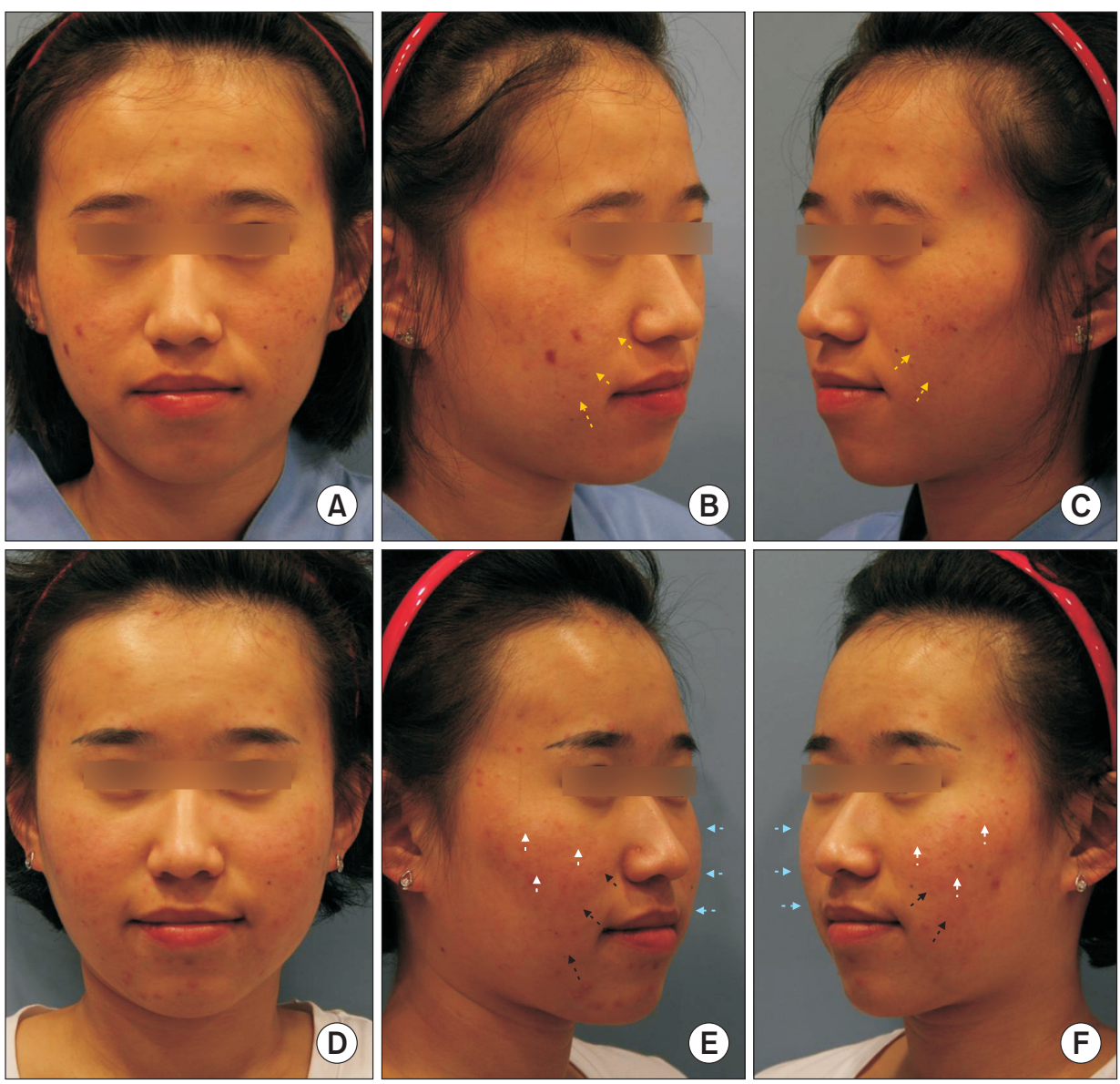

Fig. 2. A 23-year-old female who underwent sequential autologous fat injection to improve infraorbital hollowness and deep nasolabial fold (NLF) (case 2; Table 1). The amount of injected fat for the face, barring the forehead region, was approximately $43.0 \mathrm{ml}$. Despite great volume restoration, sagging is not obvious, and a balanced and lifted oval-shaped face is observed. The NLFs are softened, and the wrinkle severity rating scale grade is unchanged. Series of facial arcs and convexities with a smooth transition and improved double S-shaped Ogee curves are seen (E, F). (A-C) Preoperative photos; (D-F) postoperative photos, 1 year and 6 months after fat grafting. Yellow arrows indicate preoperative status; white arrows, postoperative volume increase; blue arrows, change of Ogee curve; and black arrows, postoperative volume decrease.
Fig. 2, 3). However, in the case of a flatter midface (cases 8-10), the anterior projection rather increased the volume in the NLFs, thereby aggravating the NLFs (cases 8-10; Fig. 4). Consequently, the midface looked more natural and lifted.

Fine, full, and tightened Ogee S-curves were postoperatively observed in 8 patients who had small and flat malar bones (cases 1-6, and 9; Fig. 2-4).

Following the SAFI technique, fat grafting to the cheek and submalar depression led jowl volume reduction and labiomandibular fold shortening (Fig. 3, 4). However, in cases with smaller chins (cases 2,6), we observed neither volume reductions nor gravitational descents (Fig. 2).

\section{Discussion}

In general, the facial fat grafting procedure is considered as a volume restoration surgery. In young patients with poorly developed midfacial skeletal systems and older patients with bony resorption with or without soft tissue sagging, fat grafting frequently causes an unnatural contour with a sagging appearance, which is an unexpected complication.
The SAFI technique was first developed to prevent fat grafting-induced sagging, and it showed a lifting phenomenon due to fat volume increase [11]. The key aspects of the SAFI technique are the order of injection (from superolateral to inferomedial regions) and the administration of different amounts of fat injection according to the layers.

We evaluated 10 young patients who underwent the SAFI technique for the correction of facial volume deficiency and sagging appearance. Despite being in their 20s, they had smaller or flatter malar bones with negative globe-to-skeletal rim relations. The common injected regions were palpebromalar groove, malar eminence, lateral part of submalar depression, tear trough deformity, mid-cheek furrow, anterior part of submalar depression (Fig. 1, region 1-11, 13-15).

In the cases of decreased WSRS grades (the right side of cases 1, 2, and 5; the left side of case 4; and both sides of cases 3, 6 , and 7), the volume reduction and unfolding of the lateral part of the NLFs, as the evidence of real volumetric face lift, appeared to be due to the increased midface volume (cases 2,5 , and 7 ). This showed similar results to those of the study wherein NLF was improved by injecting fillers into the supra-periosteal layer 

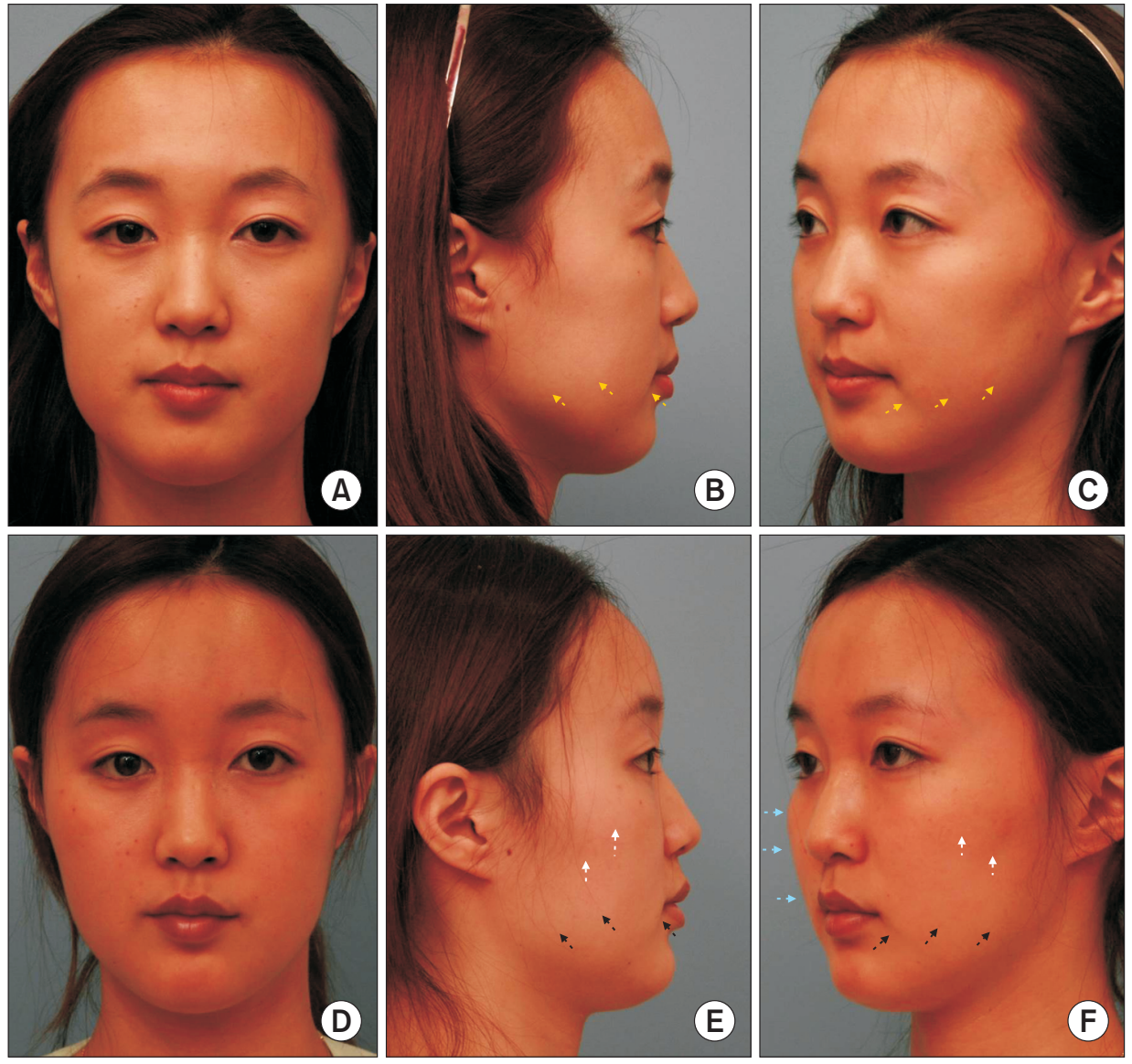

Fig. 3. A 27-year-old female who underwent sequential autologous fat injection to improve an asymmetric face due to smaller and flatter right malar bones and sagging appearance (case 4; Table 1). The amount of injected fat was approximately $31.0 \mathrm{ml}(21.0 \mathrm{ml}$ on the right side). The facial contour was changed to a symmetrical and oval shape, mainly due to great volume restoration for the projection of the midface and cheek region. Eventually, it led to nasolabial fold softening and jowl reduction. The Ogee curve significantly changed on the right side (C, F). (A-C) Preoperative photos; (D-F) postoperative photos, 6 months after fat grafting. Yellow arrows indicate preoperative status, white arrows, postoperative volume increase; blue arrows, change of Ogee curve; and black arrows, postoperative volume decrease. of the malar region [12]. This is considered as an evidence of a real volumetric facelift.

Three patients with increased WSRS grades had maxillary bone under-development (cases 7-9). Sufficient anterior projection by fat grafting in this area contributed to NLF volume increase, resulting in deep NLFs and WSRS grade increase. However, the entire midface was lifted by the grafted fat, and the volume of the folding part of the NLF was reduced. Moreover, the NLF contours appeared much more natural, which was an evidence of a real volumetric face lift (Fig. 4).

Preoperatively, the lateral malar bone was relatively well developed (Fig. 4), whereas the anterior malar bone was flat and less protruded. Severe submalar depression and sagging jowls were also combined.

Sufficient fat injection to these volume-deficient regions changed the lower facial contour from a rectangular to an oval shape with a lifted appearance, decreased the volume of the superior part of the jowl, and shortened the labiomandibular folds.

Particularly, an asymmetric face with an under-developed right malar bone caused a severe sagging appearance (Fig. 3).
Substantial volume restoration in the temple, malar region, and lateral cheek depression led to jowl volume reduction, changed the facial contour from a rectangular to an oval shape, and showed a gentle Ogee S-curve as the real evidence of volumetric face lift.

However, in 2 patients (cases 2 and 6), we observed neither an apparent jowl reduction nor a sagging appearance. This was caused by insufficient skeletal support due to a small chin, with volume loss in the pre-jowl region despite great volume restoration from the midface and cheek. Additionally, we observed insufficient volume restoration in the chin and pre-jowl regions after fat grafting (Fig. 2). In these cases, it is necessary to perform additional fat grafting, implantation, or jaw surgery to increase skeletal support for the jowl.

This study presented evidence to prove that the SAFI technique, using only fat grafting, had a volumetric lifting effect, even though the results were not statistically significant. However, to perfect the SAFI effect, the study should be conducted on a larger number of patients, with a long-term follow-up, and using advanced image devices for analysis.

The SAFI technique showed a real evidence of a volumetric 

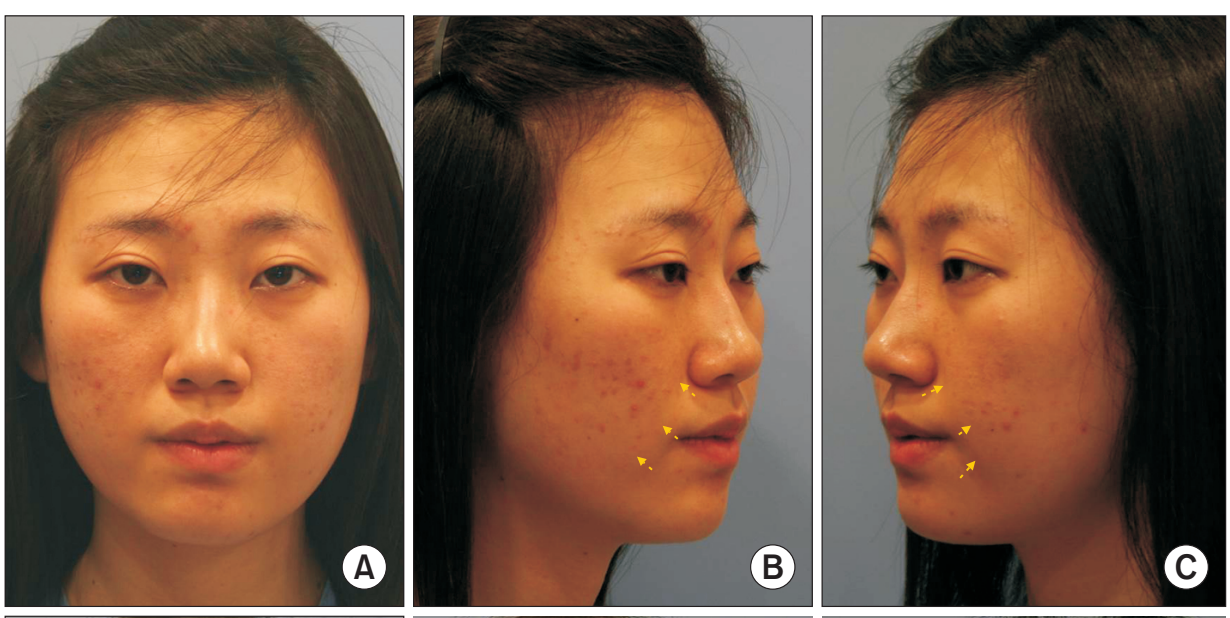

Fig. 4. A 21-year-old female who underwent sequential autologous fat injection to improve infraorbital and cheek hollowness. Upper blepharoplasty was done simultaneously (case 9; Table 1). The amount of injected fat for the face, barring the forehead region, was approxi-
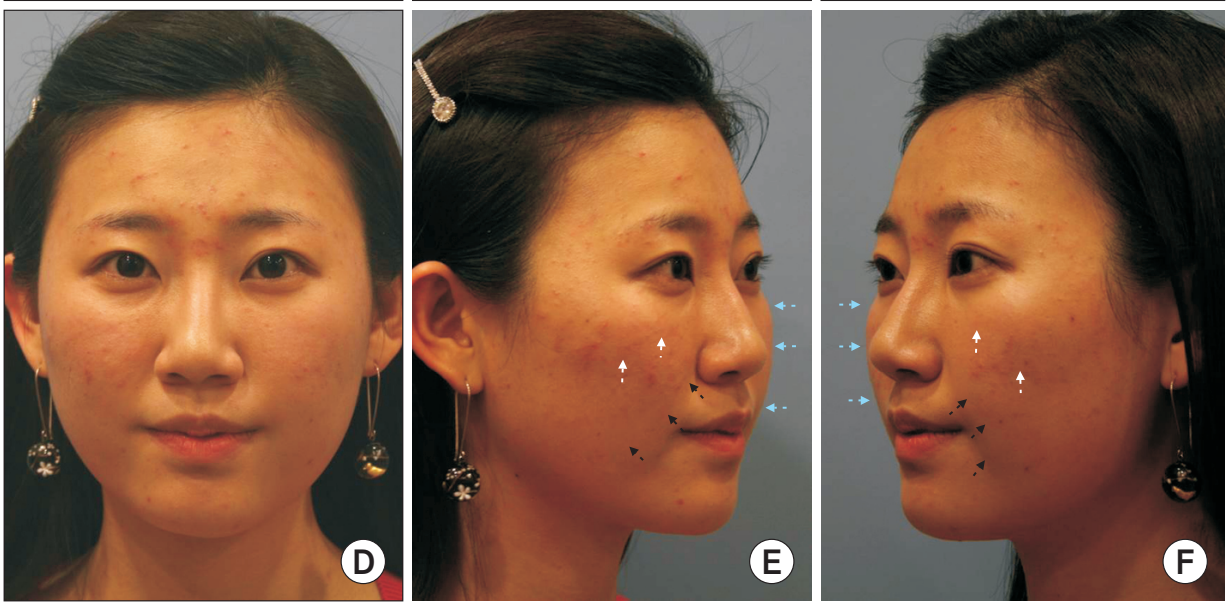

mately $40.0 \mathrm{ml}$. The facial contour was dramatically changed from a rectangle shape to a balanced oval shape due to anterior midface protrusion and jowl fat reduction. An improved Ogee S-curve with fullness due to anterior projection is observed. Interestingly, the nasolabial folds (NLFs) appear more aggravated due to a significant increase in midfacial volume, despite NLF fat reduction. (A-C) Preoperative photos; (D-F) postoperative photos, 4 months after fat grafting. Yellow arrows indicate preoperative status; white arrows, postoperative volume increase; blue arrows, change of Ogee curve; and black arrows, postoperative volume decrease.

lifting with both NLF softening and jowl volume reduction in young females with insufficient midface volume.

\section{Conflicts of interest}

The author has nothing to disclose.

\section{References}

1. Coleman SR. Structural fat grafting. Aesthet Surg J 1998;18: 386, 388.

2. Donofrio LM. Fat rebalancing: the new "facelift". Skin Therapy Lett 2002;7:7-9.

3. Amar RE, Fox DM. The facial autologous muscular injection (FAMI) procedure: an anatomically targeted deep multiplane autologous fat-grafting technique using principles of facial fat injection. Aesthetic Plast Surg 2011;35:502-10.

4. Lam SM, Glasgold MJ, Glasgold RA. Complementary fat grafting. Philadelphia, PA: Lippincott, Williams, \& Wilkins; 2007.

5. Kang K. Lifting effect of sequential autologous fat injection
(SAFI) in three patients with small and flat malar bones. J Cosmet Med 2019;3:120-4.

6. Prager W, Wissmueller E, Havermann I, Bee EK, Howell DJ, Zschocke I, et al. A prospective, split-face, randomized, comparative study of safety and 12-month longevity of three formulations of hyaluronic acid dermal filler for treatment of nasolabial folds. Dermatol Surg 2012;38(7 Pt 2):1143-50.

7. Pessa JE, Zadoo VP, Mutimer KL, Haffner C, Yuan C, DeWitt AI, et al. Relative maxillary retrusion as a natural consequence of aging: combining skeletal and soft-tissue changes into an integrated model of midfacial aging. Plast Reconstr Surg 1998;102:205-12.

8. Kang KJ, Yang HH, Chai CY. Assessment of rejuvenation by change of lid-cheek junction after transconjunctival and traditional subciliary incision blepharoplasty. J Cosmet Med 2017;1:30-8.

9. Day DJ, Littler CM, Swift RW, Gottlieb S. The wrinkle severity rating scale: a validation study. Am J Clin Dermatol 2004;5:4952.

10. Davis A, Augenstein A. Amniotic allograft implantation for 
midface aging correction: a retrospective comparative study with platelet-rich plasma. Aesthetic Plast Surg 2019;43:134552.

11. Kang KJ. SAFI: a novel facial fat grafting technique with a concept of volumetric lifting. Paju: Koonja Publishing Inc.; 2016. p.
$1-351$.

12. El-Mesidy MS, Alaklouk WT, Azzam OA. Nasolabial fold correction through cheek volume loss restoration versus thread lifting: a comparative study. Arch Dermatol Res 2020;312:47380. 